\title{
IVyF: Validez de un Instrumento de Medida de las Fortalezas del Carácter de la Clasificación de Peterson y Seligman (2004)
}

Alejandro César Cosentino ${ }^{1}$ y Alejandro Castro Solano²

\section{Artículo}

Material original autorizado para la publicación en la revista Psicodebate. Facultad de Ciencias Sociales. Universidad de Palermo.

Recibido 10-07-2015 | Aceptado 14-08-2015

\section{Resumen}

El IVyF (Strength of Character Inventory, en inglés) es un instrumento de medición de las 24 fortalezas del carácter de la clasificación de Peterson y Seligman (2004). A diferencia de otros cuestionarios homólogos para medir las fortalezas de esa clasificación que están compuestos por decenas de ítems, el IVyF es un instrumento de medición de 24 ítems. Este estudio se enfocó en la validez del IVyF. En primer lugar, el IVyF estuvo asociado a medidas de variables conceptualmente relevantes como satisfacción con la vida, los factores de personalidad del modelo de los Cinco Grandes, y deseabilidad social. En segundo lugar, se hallaron asociaciones con tamaños del efecto mayores a mediano entre autopuntuación y puntuación realizada por un observador. Los resultados de esta investigación han sido similares a los obtenidos con cuestionarios homólogos. Se concluye que el IVyF presenta no sólo adecuada confiabilidad, sino también adecuada validez.

Palabras Clave: psicología positiva, virtudes, test, validez.

1 Universidad de Palermo - Argentina; acosentino@outlook.com 


\section{Abstract}

The Strengths of Character Inventory (SCI; IVyF in Spanish) is a measurement instrument for the 24 character strengths from the Peterson and Seligman (2004) character classification. Unlike other homologous questionnaires to measure the character strengths of this classification that are composed of dozens of items, the SCI is constituted by 24 items. This study focused on the validity of the SCI. Firstly, the SCI was associated with measures of conceptually relevant variables such as life satisfaction, personality from the Big Five model, and social desirability. Secondly, associations with more than medium effect size were found between self-rating and observer's rating. The results of this research were similar to those obtained with homologous questionnaires. We conclude that SCI presents not only acceptable reliability, but also acceptable validity.

Keywords: positive psychology, virtues, test, validity. 
La Psicología Positiva puede considerarse constituida por tres áreas o pilares: la subjetiva, la individual y la grupal (Gable \& Haidt, 2005; Seligman \& Csikszentmihalyi, 2000). El área subjetiva se relaciona con el estudio de las experiencias subjetivas valoradas positivamente, el área individual, que estudia los rasgos individuales positivos, como la capacidad de amar, el coraje, las habilidades interpersonales, la sensibilidad estética, la perseverancia, la clemencia, la originalidad, la espiritualidad y la sabiduría; en resumen, las virtudes y fortalezas del carácter, y finalmente, en el área grupal se investiga a los grupos humanos en relación con los aspectos positivos de los individuos, por ejemplo, a las instituciones que impulsan a los individuos a ser mejores ciudadanos.

Peterson y Seligman (2004) impulsaron el estudio de los rasgos individuales positivos o fortalezas del carácter con una metodología científica. Estos autores han considerado que contar con una clasificación de las virtudes y fortalezas del carácter constituía un paso importante y necesario para el progreso del estudio científico en Psicología Positiva, y se tomaron la tarea de desarrollar esa clasificación.

El paso inicial en el camino de desarrollo de su clasificación (Dahlsgaard, Peterson, \& Seligman, 2005), incluyó el examen de las respuestas sobre el comportamiento moralmente bueno que se concibieron en las tradiciones filosóficas y religiosas de impacto evidente y duradero en la civilización humana: el confucianismo y taoísmo (de China), el budismo e hinduismo (del sur asiático), y la filosofía ateniense, el judaísmo, el cristianismo y el islamismo (de Occidente). Seis virtudes fundamentales se repetían en esas tradiciones y esta convergencia sugirió un fundamento no arbitrario para la nueva clasificación, evitando que presentase un sesgo histórico o cultural. A fin de generar las fortalezas del carácter para incluir en la clasificación, un grupo de académicos propuso una lista de fortalezas candidatas que fue refinada a través de una serie de debates llegando a una clasificación de 6 virtudes que incluyó 24 fortalezas (Peterson \& Seligman, 2004).

El carácter en la clasificación de Peterson y Seligman (2004) incluye las seis virtudes (las fortalezas del carácter correspondientes aparecen entre paréntesis): coraje (integridad, persistencia, valentía, vitalidad), justicia (imparcialidad, compañerismo y liderazgo), humanidad (amor, bondad, inteligencia social), sabiduría y conocimiento (apertura mental, amor por el saber, creatividad, curiosidad, perspectiva), templanza (autorregulación, clemencia, humildad, y prudencia) y trascendencia (admiración de la belleza y la excelencia, esperanza, espiritualidad, gratitud y humor). No obstante, Peterson y Seligman afirmaron que esta clasificación era de carácter tentativo y que podría ser alterada a consecuencia del progreso en el estudio científico. 


\section{Instrumentos para la medición de las fortalezas del carácter de la clasificación de Peterson y Seligman (2004)}

Hay varios instrumentos que pueden utilizarse para medir las 24 fortalezas del carácter de la clasificación de Peterson y Seligman (2004) para población adulta. Algunos de ellos se indican abajo.

\section{Values in Action Inventory of Strengths (VIA-IS)}

Peterson y Seligman (2004) han desarrollado el VIA-IS que es un cuestionario de autoinforme de 240 ítems para evaluar las 24 fortalezas del carácter según la clasificación Values in Action (VIA) en población adulta. Este cuestionario puede completarse libremente online desde fines de 2001 - p. ej., http://www. viacharacter.org-, y hay también una versión de VIA-IS en formato de papel y lápiz (Linley et al., 2007). Además, hay versiones disponibles del VIA-IS en español y en otros idiomas, y se continúan desarrollando versiones en varios idiomas más. Cuando se administra el VIA-IS, el entrevistado indica con cuánta frecuencia se percibe a sí mismo teniendo estados representativos de las fortalezas del carácter, usando una escala Likert de cinco puntos que va de muy parecido a mí a muy diferente a mí.

\section{International Personality Item Pool VIA revisado (IPIP-VIA-r)}

El International Personality Item Pool (IPIP) es un emprendimiento de colaboración científica para el desarrollo de mediciones de la personalidad y otras diferencias individuales que ha sido presentado por primera vez en 1996 en la octava European Conference on Personality (Goldberg, 1999; Goldberg et al., 2006). Su sitio web (http://ipip.ori.org) está destinado a proporcionar acceso rápido a mediciones de diferencias individuales de dominio público, i. e., sin copyright. Desde el sitio web del IPIP, pueden obtenerse los ítems en inglés constituyentes del instrumento IPIP-VIA-r para evaluar las 24 fortalezas del carácter siguiendo la clasificación VIA. El sitio provee 213 ítems a los fines de evaluar las 24 fortalezas del carácter de la clasificación VIA. Ejemplos de ítems — que se tradujeron al español— del IPIP-VIA-r son: suelo obtener lo que quiero porque trabajo mucho para conseguirlo —-fortaleza persistencia-, me pongo a pensar antes de hablar - fortaleza prudencia-, me surgen ideas nuevas y diferentes - fortaleza creatividad-.

\section{IVyF}

El IVyF (Cosentino \& Castro Solano, 2008b) es un inventario de ítems de párrafo bipolares de autopuntuación directa global para evaluar las 24 fortalezas 
del carácter de la clasificación de Peterson y Seligman (2004). El instrumento consta de 24 ítems bipolares, correspondiendo cada ítem a una fortaleza, los cuales se responden con una escala tipo Likert que va de 1 -Soy muy parecido a la primera persona - a 5 - Soy muy parecido a la segunda persona- Los ítems $2,3,5,8,9,12,14,15,17,20,22$ y 23, tienen puntuación directa; en tanto que tienen puntuación revertida los ítems 1, 4, 6, 7, 10, 11, 13, 16, 18, 19, 21, 24. (El IVyF puede ser solicitado a sus autores a fin de que pueda ser utilizado en investigaciones académicas, y será enviado en su formato digital.)

Se incluye un ejemplo de ítem gratitud del IVyF. El polo ausencia de la fortaleza del carácter gratitud está construido por el párrafo: "Son pocas las cosas por las cuales pueda sentirme agradecido. No me veo a mi mismo como una persona afortunada porque no creo que haya sido ricamente bendecido en la vida. Así que, evidentemente, no tengo todos los días un profundo sentimiento de gratitud por lo que me tocó vivir. Además, no siento la necesidad de darle las gracias ni le demuestro mi agradecimiento a la gente que es buena conmigo y que se preocupa por mí.". El polo de presencia de gratitud está constituido por el siguiente párrafo: "No son pocas las cosas por las cuales puedo sentirme agradecido. Me veo a mi mismo como una persona afortunada porque creo que he sido ricamente bendecido en la vida. Así que, evidentemente, tengo todos los días un profundo sentimiento de gratitud por lo que me tocó vivir. Además, siento la necesidad de darle las gracias y le demuestro mi agradecimiento a la gente que es buena conmigo y que se preocupa por mí.".

Se han realizado algunos análisis psicométricos sobre el IVyF. Cosentino (2009) ha mostrado que tomando como punto de referencia cuatro análisis psicométricos sobre puntuaciones producidas por instrumentos que evalúan la clasificación VIA (Macdonald, Bore, \& Munro, 2008; Peterson \& Seligman, 2004; Peterson, Park, Pole, D'Andrea, \& Seligman, 2008; “IPIP”), las confiabilidades y la validez factorial del IVyF guardan similitud.

Por una parte, el IVyF tiene una aceptable confiabilidad test-retest, (aproximadamente 3 semanas de separación temporal entre administraciones), con rs dentro del rango de 72-.92, $M=.80$, y el alfa de Cronbach sobre las puntuaciones de los 24 ítems individuales, considerando a este alfa como un indicador del grado en que están interrelacionados - i. e., co-varían - las respuestas a los ítems dadas por los participantes (Helms et al., 2006) fue de $\alpha=.85$ (Cosentino, 2009). La confiabilidad de tipo estabilidad del IVyF es aceptable y semejante a la hallada para el VIA-IS (Peterson \& Seligman, 2004; Ruch et al., 2010).

Por otra parte, la cantidad y composición de los factores del IVyF es similar a los factores informados por Peterson y Seligman (2004), que corresponden a fortalezas interpersonales, fortalezas intelectuales, fortalezas de la restricción, 
fortalezas emocionales y fortalezas teológicas (Cosentino, 2009). Sin embargo, debe ser destacado que la estructura factorial de la clasificación VIA es controversial, ya que el resultado de varios análisis factoriales han mostrado variaciones en la composición y cantidad de los factores extraídos, que generalizadamente no coinciden con la cantidad y composición de los factores teóricamente propuestos (e.g., Peterson \& Seligman, 2004; Peterson et al., 2008; Macdonald et al., 2008; Ruch et al., 2010).

\section{La presente investigación}

El objetivo de esta investigación es mostrar análisis psicométricos del IVyF haciendo foco en diferentes tipos de validez. Tres relaciones son fundamentales para establecer la validez de este nuevo instrumento basado en las relaciones teóricas y empíricas entre satisfacción vital, personalidad y deseabilidad social. Respecto de la satisfacción con la vida, se ha propuesto que, por definición, las fortalezas del carácter contribuyen a la realización, la satisfacción y la felicidad personal en un sentido amplio (Park, Peterson, \& Seligman, 2004; Peterson \& Seligman, 2004). Respecto de la personalidad, Peterson y Seligman han afirmado que es substancial la existencia de relaciones entre su clasificación del carácter y el modelo de la personalidad Big Five, desarrollado a partir de la convergencia en factores o dimensiones de las palabras referidas a los rasgos que se hallan en el lenguaje (Goldberg, 1993), ya que hace que su clasificación tenga sentido. Finalmente, Peterson y Seligman han afirmado que las fortalezas del carácter son socialmente deseables.

Adicionalmente, se estudió la validez del IVyF en relación con un criterio objetivo y en relación a un cuestionario que evalúa fortalezas del carácter.

\section{Estudio 1: Relaciones del IVyF con una Medición de Satisfacción Vital}

Park et al. (2004) han hallado que las fortalezas están asociadas con la satisfacción subjetiva con la vida, y que las fortalezas del carácter esperanza, vitalidad, gratitud, amor y curiosidad son las que presentan asociaciones más altas con esa variable. Estos autores sugirieron que no se podría afirmar que el carácter bueno provoca el buen vivir, sino más bien que la satisfacción con la vida podría considerarse como un aspecto inherente al buen carácter, del mismo modo que la gracia es inherente a bailar bien y no su resultado o efecto. En consecuencia, se espera que las puntuaciones del IVyF se asocien con las puntuaciones de satisfacción vital. 


\section{Participantes}

La muestra de participantes de Argentina estuvo integrada por 168 varones y 172 mujeres, $n=340$, con una media de edad de 33.8 años, DT $=12.5$, quienes fueron voluntarios que no recibieron retribución alguna por su participación.

\section{Instrumentos}

$I V y F$. Se utilizó un inventario de papel y lápiz compuesto por 24 ítems de autopuntuación directa global que evalúan las 24 fortalezas del carácter según la clasificación VIA de Peterson y Seligman (2004). La confiabilidad de tipo consistencia interna del IVyF en esta muestra fue de $\alpha=.84$, utilizando alfa de Cronbach, considerando a este alfa como un indicador del grado en que están interrelacionados - i. e., covarían - las respuestas a los ítems dadas por los participantes (Helms et al., 2006).

Satisfaction with Life Scale (SWLS). Para medir el constructo satisfacción con la vida se utilizó la adaptación de la escala SWLS (Castro Solano, 2000; Diener, Emmons, Larsen, \& Griffin, 1985) que evalúa el componente cognitivo del bienestar. El instrumento está compuesto por 5 ítems que se responden por medio de una escala tipo Likert que va de 1 - muy en desacuerdo - a 7 - muy de acuerdo -; el inventario produce una puntuación total, correspondiendo las puntuaciones más elevadas a mayor satisfacción con la vida. La consistencia interna de la SWLS para la muestra de este estudio es de $\alpha=.84$.

\section{Procedimiento y análisis de datos}

Los participantes completaron el IVyF y la SWLS en su formato de papel y lápiz. El análisis de datos se realizó por medio de la obtención de coeficientes de correlación simple entre las puntuaciones del IVyF y de la SWLS, y de coeficientes de correlación parcial entre las puntuaciones de esos instrumentos, mientras se controlaban los efectos de las variables sexo y edad.

\section{Resultados}

Todas las fortalezas del carácter se asociaron con satisfacción con la vida de forma estadísticamente significativa - exceptuando humildad - y directa. Gratitud, curiosidad, vitalidad, esperanza, persistencia y amor presentaron asociaciones de tamaño superior a mediano con satisfacción con la vida, y estas relaciones se mantuvieron al controlar las variables edad y sexo; ver la Tabla 1. 
Tabla 1.

Correlaciones entre Fortalezas del Carácter y Satisfacción con la Vida

\begin{tabular}{|c|c|c|}
\hline Fortaleza & SWLSa & $S W L S b$ \\
\hline Gratitud & $.56^{\star \star}$ & $.57^{\star \star}$ \\
\hline Curiosidad & $.46^{\star \star}$ & $.46^{\star \star}$ \\
\hline Vitalidad & $.44^{\star \star}$ & $.44^{\star \star}$ \\
\hline Esperanza & $.40^{\star \star}$ & $.40^{\star \star}$ \\
\hline Persistencia & $.33^{\star *}$ & $.33^{\star *}$ \\
\hline Amor & $.31^{* *}$ & $.32^{* *}$ \\
\hline Bondad & $.28^{\star \star}$ & $.29^{\star \star}$ \\
\hline Perspectiva & $.30^{\star \star}$ & $.29^{\star \star}$ \\
\hline Liderazgo & $.28^{* *}$ & $.28^{* *}$ \\
\hline Autorregulación & $.26^{\star \star}$ & $.26^{\star \star}$ \\
\hline Valentía & $.26^{\star \star}$ & $.26^{\star \star}$ \\
\hline Integridad & $.24^{\star \star}$ & $.25^{\star \star}$ \\
\hline Creatividad & $.24^{* *}$ & $.24^{\star \star}$ \\
\hline Apertura mental & $.21^{\star \star}$ & $.21^{\star \star}$ \\
\hline Amor por saber & $.21^{\star \star}$ & $.21^{\star \star}$ \\
\hline Apreciación & $.19^{\star \star}$ & $.20^{\star \star}$ \\
\hline Inteligencia social & $.19^{\star \star}$ & $.19^{\star \star}$ \\
\hline Ciudadanía & $.15^{\star \star}$ & $.16^{\star \star}$ \\
\hline Prudencia & $.16^{\star \star}$ & $.16^{\star \star}$ \\
\hline Espiritualidad & $.14^{\star \star}$ & $.15^{\star}$ \\
\hline Humor & $.14^{\star \star}$ & $.15^{\star}$ \\
\hline Imparcialidad & $.14^{*}$ & $.14^{*}$ \\
\hline Clemencia & $.13^{*}$ & $.13^{*}$ \\
\hline Humildad & -.07 & -.07 \\
\hline \multicolumn{3}{|c|}{$\begin{array}{l}\text { Nota. SWLS = Satisfaction With Life Scale. Apreciación = apreciación de la belleza y la } \\
\text { excelencia. } \\
{ }^{a} \text { Correlación simple con el IVyF, } n=340 \text {. } \\
{ }^{\circ} \text { Correlación parcial controlando sexo y edad con el IVyF, } n=340 . \\
{ }^{*} p<.05 \text {, bilateral. } \\
{ }^{*} p<.01 \text {, bilateral. }\end{array}$} \\
\hline
\end{tabular}




\section{Estudio 2: Relaciones del IVyF con una Medición del Modelo de Personalidad de Cinco Factores}

El modelo de personalidad de cinco factores corresponde al procedimiento léxico - lexical approach, en inglés - que se desarrolló a partir de la convergencia en factores o dimensiones de las palabras referidas a los rasgos que se hallan en el lenguaje (Goldberg, 1993). Peterson y Seligman consideran que debido a que existen en el léxico una gran cantidad de términos referidos a los rasgos que se aplican tanto a las características moralmente valoradas como a las características de personalidad, no debería resultar sorprendente encontrar vinculaciones entre la clasificación del carácter y los factores del Big Five.

Peterson y Seligman (2004) han elaborado dos propuestas en relación con el vínculo entre la personalidad según el Big Five y los rasgos del carácter. Ambas propuestas no se fundamentan en análisis empíricos de las relaciones entre de ambos constructos.

Siguiendo la primera propuesta de Peterson y Seligman (2004), los factores del Big Five que se corresponden con las fortalezas del carácter de la clasificación VIA son: a) responsabilidad con autorregulación y persistencia, b) agradabilidad con bondad y gratitud, c) apertura a la experiencia con curiosidad, creatividad y apreciación de la belleza, y d) extraversión con vitalidad y humor; en tanto el factor neuroticismo no se corresponde con ninguna fortaleza. También reconocen que los individuos esperanzados se puntúan bajo en neuroticismo y que los líderes a menudo se puntúan alto en extraversión.

Siguiendo la segunda propuesta de Peterson y Seligman, en que se correspondieron racionalmente los factores del Big Five con los agrupamientos de fortalezas del carácter extraídos por un análisis factorial exploratorio, los factores de personalidad se corresponderían con el carácter del siguiente modo: a) el factor responsabilidad del Big Five con las fortalezas de la restricción, b) agradabilidad con las fortalezas interpersonales, c) apertura a la experiencia con las fortalezas intelectuales, y d) el opuesto al neuroticismo — estabilidad emocional — con las fortalezas emocionales-. Finalmente, los autores de la clasificación VIA no vincularon ninguna fortaleza del carácter al factor extraversión del Big Five así como tampoco vincularon ninguna dimensión de la personalidad al quinto grupo de fortalezas extraído en el mencionado análisis factorial, las fortalezas teológicas.

Hay un elemento en común general entre ambas propuestas de la relación entre el Big Five y las fortalezas de la clasificación del carácter de Peterson y Seligman (2004), que es que cada factor del Big Five se corresponde biunívocamente con una fortaleza o grupo de fortalezas. Sin embargo, hay varias diferencias puntuales entre la primera y la segunda propuesta acerca de la relación entre el Big Five y las fortalezas del carácter: 
en la primera propuesta la autorregulación se vincula a la dimensión responsabilidad del Big Five, pero en la segunda a neuroticismo; humor se vincula a extraversión en la primera, a agradabilidad en la segunda; vitalidad se vincula a extraversión en la primera, a neuroticismo en la otra; se menciona que los líderes puntúan alto en extraversión, pero en la segunda propuesta liderazgo aparece vinculado a agradabilidad. Suponemos que la evidente falta de exactitud entre las propuestas de Peterson y Seligman sobre la relación entre las fortalezas y el Big Five se debe, en parte, a que sus propuestas se limitaron a mostrar ciertas correspondencias que parecían racionalmente satisfactorias; dicho de otro modo, sus propuestas no están basadas o corroboradas por un análisis empírico de la relación entre personalidad y carácter.

Sin embargo, también hay convergencias en fortalezas del carácter entre las dos propuestas de Peterson y Seligman (2004): creatividad, curiosidad y apreciación de la belleza y la excelencia se vinculan al factor apertura a la experiencia; bondad al factor agradabilidad; y también se podría llegar a considerar esperanza vinculada al factor neuroticismo —o su inverso, estabilidad emocional—a pesar de no estar explícitamente presente en la primera propuesta.

Peterson y Park (2004) han informado fragmentariamente -i. e., sin brindar información detallada que permita la replicación de la investigación, como composición muestral, momento y forma de administración, etc.- resultados empíricos sobre la relación entre las fortalezas del carácter y el NEO-PI, un instrumento para evaluar el Big Five. Debido a que brinda algunos datos empíricos relevantes, a diferencia de las otras propuestas teóricas, se incluye el tamaño del efecto de los coeficientes de correlación (Cohen, 1992) como indicador del grado de asociación entre las variables: el factor apertura a la experiencia se asocia con las fortalezas del carácter apreciación de la belleza y la excelencia, curiosidad y amor por el saber con tamaños del efecto mayores a grandes; el factor agradabilidad con trabajo en equipo, con tamaño del efecto mediano a grande; y el factor responsabilidad con persistencia, y autorregulación, con tamaños del efecto mayores a grandes.

Macdonald et al. (2008) informan de su estudio empírico desarrollado para verificar las relaciones entre los rasgos positivos del carácter de la clasificación VIA y las dimensiones de la personalidad según el Big Five. Entre otros hallazgos, se confirma la idea de Peterson y Seligman (2004) de que existen vinculaciones entre el Big Five y las fortalezas del carácter. El estudio empírico de Macdonald et al. halló que los factores del Big Five están relacionados con varias fortalezas del carácter, específicamente, el factor extraversión con 13 fortalezas, responsabilidad con 10, apertura a la experiencia con 8, agradabilidad con 6 y neuroticismo con 2. No obstante, Macdonald et al. hallaron que hubo más de un predictor del Big Five para la mayoría de las fortalezas del carácter, lo cual, según estos autores, pondría en duda la propuesta de Peterson y Seligman que vincula cada fortaleza del carácter con un solo constructo del Big Five. 


\section{Participantes}

En este estudio hubo 816 participantes de Argentina, de los cuales 505 eran mujeres y 311 eran varones, con una media de edad de 31.4 años, DT $=14.1$, los cuales aceptaron voluntariamente completar los instrumentos. Los participantes no recibieron retribución alguna por su inclusión en el estudio.

\section{Instrumentos}

$I V y F$. Se utilizó el IVyF para evaluar las fortalezas del carácter según las definiciones y clasificación de Peterson y Seligman (2004). La confiabilidad del tipo consistencia interna para esta muestra fue de $\alpha=.79$.

Big Five Inventory (BFI). Se utilizó la adaptación argentina (Castro Solano \& Casullo, 2001) para medir las dimensiones del Big Five. El BFI, que fue desarrollado por O. P. John para operacionalizar el modelo de los Cinco Grandes. El BFI mide los factores apertura a la experiencia, neuroticismo, extraversión, responsabilidad y agradabilidad. Consta de 44 ítems que se responden según una escala de 5 opciones de respuesta, que va de 1 - completo desacuerdo - a 5 - completo acuerdo-. Alos ítems los antecede una afirmación general Yo me veo a mi mismo/a como alguien... que se combina con la afirmación de cada ítem a fin de generar cada oración a ser respondida. Ejemplos de ítems son: a quien le gusta hablar o que es curioso/a respecto a las cosas. Por sus estudios de confiabilidad y validez, se concluye que el BFI es una medida válida y confiable. En esta muestra, para cada factor, las alfas de Cronbach fueron: extroversión, .72, agradabilidad, .69, responsabilidad, .77, neuroticismo, .74 , apertura a la experiencia, .79 , respectivamente.

\section{Procedimiento y análisis de datos}

Los participantes completaron ambos instrumentos, IVyF y BFI, en formato de papel y lápiz. Los análisis de datos se realizaron correlacionando, mediante $\mathrm{r}$ de Pearson, las puntuaciones de las fortalezas del carácter medidas con el IVyF con las puntuaciones de las dimensiones de la personalidad del Big Five medida con el BFI. La significación estadística se consideró de modo bilateral.

\section{Resultados}

En la Tabla 2 se muestran las correlaciones entre las puntuaciones de las fortalezas del carácter medidas con el IVyF y las puntuaciones de las dimensiones de la personalidad según el modelo de los Cinco Factores medida con el BFI. Los análisis empíricos muestran que los rasgos de personalidad están asociados a las fortalezas del carácter. 
Tabla 2.

Correlaciones entre Fortalezas del Carácter y Dimensiones de la Personalidad

\begin{tabular}{|c|c|c|c|c|c|}
\hline & $R$ & $A$ & $A E$ & $N$ & $E$ \\
\hline Amor & $.09^{* *}$ & $.22^{\star \star}$ & .07 & -.02 & $.24^{\star *}$ \\
\hline Amor por saber & $.15^{\star \star}$ & $.08^{\star}$ & $.35^{\star \star}$ & $-.11^{\star \star}$ & .05 \\
\hline Apertura Mental & $.27^{\star \star}$ & $.12^{\star \star}$ & $.14^{\star \star}$ & $-.15^{\star \star}$ & $-.08^{\star}$ \\
\hline Apreciación & $.18^{\star \star}$ & $.16^{\star \star}$ & $.35^{\star \star}$ & -.06 & $.14^{\star \star}$ \\
\hline Autorregulación & $.34^{\star \star}$ & $.12^{\star \star}$ & .06 & $-.17^{\star \star}$ & -.00 \\
\hline Bondad & $.18^{\star \star}$ & $.47^{\star \star}$ & $.13^{\star \star}$ & $-.08^{\star}$ & $.16^{\star \star}$ \\
\hline Ciudadanía & $.12^{\star \star}$ & $.21^{\star \star}$ & .04 & $-.09^{\star \star}$ & $.19^{\star \star}$ \\
\hline Clemencia & $.12^{\star \star}$ & $.38^{\star \star}$ & $.13^{\star \star}$ & $-.23^{\star \star}$ & .00 \\
\hline Creatividad & $.09^{\star}$ & .05 & $.57^{\star *}$ & $-.13^{\star \star}$ & $.18^{\star \star}$ \\
\hline Curiosidad & $.21^{\star \star}$ & $.18^{\star \star}$ & $.29^{\star \star}$ & $-.25^{\star \star}$ & $.16^{\star \star}$ \\
\hline Esperanza & $.32^{\star \star}$ & $.22^{\star \star}$ & $.18^{\star \star}$ & $-.36^{\star *}$ & $.13^{\star \star}$ \\
\hline Espiritualidad & $.24^{\star \star}$ & $.20^{\star \star}$ & .03 & -.03 & .04 \\
\hline Gratitud & $.22^{\star \star}$ & $.29^{\star \star}$ & $.12^{\star \star}$ & $-.21^{\star \star}$ & $.15^{\star \star}$ \\
\hline Humildad & $.14^{\star \star}$ & $.25^{\star \star}$ & $-.16^{\star \star}$ & $-.09^{\star}$ & $-.29^{\star \star}$ \\
\hline Humor & -.05 & $.10^{\star \star}$ & $.18^{\star \star}$ & $-.16^{\star \star}$ & $.36^{\star *}$ \\
\hline Imparcialidad & $.12^{\star \star}$ & $.41^{\star \star}$ & $.15^{\star \star}$ & $-.21^{\star \star}$ & .02 \\
\hline Integridad & $.31^{\star *}$ & $.28^{\star *}$ & .07 & $-.14^{\star \star}$ & -.04 \\
\hline Inteligencia Social & $.12^{\star \star}$ & $.15^{\star \star}$ & $.18^{\star \star}$ & $-.19^{\star \star}$ & $.48^{\star \star}$ \\
\hline Liderazgo & $.19^{\star \star}$ & $.17^{\star \star}$ & $.19^{\star \star}$ & $-.16^{\star \star}$ & $.25^{\star \star}$ \\
\hline Persistencia & $.59^{\star \star}$ & $.14^{\star \star}$ & .06 & $-.12^{\star \star}$ & $.07^{*}$ \\
\hline Perspectiva & $.19^{\star \star}$ & $.22^{\star \star}$ & $.20^{\star \star}$ & $-.22^{\star \star}$ & $.10^{\star *}$ \\
\hline Prudencia & $.29^{\star \star}$ & $.32^{\star *}$ & .01 & $-.17^{\star \star}$ & $-.11^{\star \star}$ \\
\hline Valentía & $.13^{\star \star}$ & -.01 & $.21^{\star \star}$ & -.07 & $.30^{\star \star}$ \\
\hline Vitalidad & $.36^{\star \star}$ & $.21^{\star \star}$ & $.18^{\star \star}$ & $-.28^{\star \star}$ & $.26^{\star \star}$ \\
\hline
\end{tabular}

Nota. Se destacan en negrita las correlaciones con tamaños del efecto superiores a mediano ( $r$ =.30; Cohen, 1992). Apreciación = apreciación de la belleza y la excelencia; $R=$ responsabilidad; $\mathrm{A}$. = agradabilidad; $\mathrm{AE}=$ apertura a la experiencia; $\mathrm{N}=$ neuroticismo; $\mathrm{E}$ = extraversión.

${ }^{*} p<.05$, bilateral.

${ }^{* *} p<.01$, bilateral. 
Resumidamente, se presentan a continuación los resultados hallados en este estudio - entre guiones se detallan las fortalezas del carácter que se asociaron con un tamaño del efecto mínimamente mediano (Cohen, 1992) con los factores de los Cinco Grandes, ordenadas en sucesión de modo que estén primero las fortalezas del carácter que tuvieron un tamaño del efecto más elevado-. Fueron directas: todas las asociaciones de las fortalezas del carácter con el factor responsabilidad - persistencia, vitalidad, autorregulación, esperanza, integridad-, todas las asociaciones con el factor agradabilidad - bondad, imparcialidad, clemencia, prudencia-, casi todas las asociaciones con el factor apertura a la experiencia - creatividad, amor por el saber, y apreciación de la belleza y la excelencia—, y la mayoría de las asociaciones con el factor extraversión $\neg$-inteligencia social, humor y valentía. Al contrario, fueron inversas todas las asociaciones halladas entre las fortalezas del carácter y la dimensión neuroticismo - esperanza-.

\section{Estudio 3: Relaciones del IVyF con una Medición de Deseabilidad Social}

La deseabilidad social hace referencia a que los individuos responden (p. ej., a un cuestionario o en una entrevista) de un modo culturalmente apropiado y aceptable debido a su necesidad de obtener aceptación o aprobación de los demás (Crowne \& Marlowe, 1964). En consecuencia, cuando los individuos se atribuyen características culturalmente apropiadas y aceptables - i. e., socialmente deseables-, se conjetura la vinculación de sus respuestas con la deseabilidad social.

Peterson y Seligman (2004) han afirmado que las fortalezas del carácter son socialmente deseables. Esta afirmación parecería llevar a la consecuencia lógica de que los investigadores deberían buscar y hallar correlaciones entre las fortalezas y la deseabilidad social. Contrariamente, los autores de la clasificación VIA han sostenido que las fortalezas del carácter no correlacionan con la deseabilidad social. Peterson y Seligman han mantenido esa afirmación sobre un extracto de resultados -i. e., resultados que no muestran información detallada que habilite la replicación del estudio—, mostrando a modo de excepción que solo dos fortalezas del carácter presentaron asociaciones con deseabilidad social—las cuales, sin embargo, presentaban una magnitud de correlación mediana o mayor a mediana entre esos constructos (Cohen, 1992).

Se propone que hay un cierto grado de inconsistencia en las propuestas de Peterson y Seligman (2004) sobre la vinculación entre las fortalezas del carácter y la deseabilidad social. Parecería más conveniente suponer que si, por definición, las fortalezas del carácter son socialmente deseables, entonces las fortalezas deberían tender, en general, a mostrarse empíricamente correlacionadas con deseabilidad social. Esta conclusión, que se considera más parsimoniosa que la de 
Peterson y Seligman, puede apoyarse empíricamente en lo hallado tanto por dos investigaciones empíricas independientes que mostraron que más de la mitad de los rasgos de carácter presentan asociaciones con la deseabilidad social (Macdonald et al., 2008; Sarros \& Cooper, 2006), como por una investigación realizada con adolescentes que halló un resultado semejante (Osin, 2009). En otro estudio se ha hallado que más de un tercio de las fortalezas correlacionaban con las puntuaciones de una escala que fue usada para evaluar deseabilidad social (Ruch et al., 2010).

Por lo tanto, se considera que la propuesta que han sostenido los autores de la clasificación VIA con relación a la vinculación entre las fortalezas del carácter y la deseabilidad social es criticable y no se halla apoyo sobre los hallazgos empíricos más recientes.

\section{Participantes}

Esta muestra estuvo compuesta por 737 participantes de Argentina de los cuales 310 eran varones y 427 eran mujeres. La media de edad fue de 40.5 años, $D T=16.5$.

\section{Instrumentos}

$I V y F$. Se utilizó el IVyF para evaluar las fortalezas del carácter según las definiciones y clasificación de Peterson y Seligman (2004).

Escala de Deseabilidad Social de Marlowe y Crowne (EDSCM). Para evaluar la deseabilidad social se utilizó la EDSCM (Cosentino \& Castro Solano, 2008a). La EDSCM es una adaptación para población argentina de la escala completa Marlowe-Crowne Social Desirability Scale, que es un autoinforme desarrollado para evaluar deseabilidad social independientemente de psicopatología, en su formato original de papel y lápiz. Consta de 33 ítems que se contestan por verdadero $(\mathrm{V})$ o falso $(\mathrm{F})$. La EDSCM produce una sola puntuación total que se obtiene de sumar las puntuaciones de cada ítem, debiéndose previamente invertir la puntuación de los ítems de puntuación revertida. A mayor puntuación, mayor deseabilidad social. La EDSCM cuenta con adecuada consistencia interna, validez con relación a la escala $L$ del Eysenck Personality Questionnaire, validez divergente con la segunda edición del Inventario de Depresión de Beck, validez de instrucciones diferenciales; y validez de grupos conocidos $\neg$ (Cosentino \& Castro Solano). La consistencia interna para las puntuaciones del EDSCM de la muestra del presente análisis fue de $\alpha=.84$.

\section{Procedimiento y análisis de datos}

Los participantes completaron los instrumentos IVyF y la EDSCM, ambos en su formato de papel y lápiz. Los análisis de datos se realizaron correlacionando, mediante $r$ de Pearson, las puntuaciones del IVyF, que mide fortalezas del carácter, 
con las puntuaciones de la EDSCM, que mide deseabilidad social independiente de psicopatología. La significación estadística se consideró de modo bilateral.

\section{Resultados}

Todas las fortalezas del carácter medidas con el IVyF se asociaron de forma positiva y estadísticamente significativa con deseabilidad social evaluada a través del EDSCM (Ver Tabla 3). Los resultados de este estudio han resultado similares a los hallados en la investigación de Macdonald et al. (2008) con una muestra de 123 estudiantes universitarios que completaron el IPIP-VIA para medir fortalezas del carácter, y una versión abreviada de la escala de deseabilidad social de Marlowe y Crowne. Es particularmente coincidente entre ambas investigaciones, que clemencia e imparcialidad presentaron las más elevadas correlaciones con deseabilidad social.

\section{Tabla 3.}

Correlaciones entre Fortalezas del Carácter y Deseabilidad Social

\begin{tabular}{|c|c|}
\hline Variable & EDSCM \\
\hline Clemencia & $.39^{\star *}$ \\
\hline Imparcialidad & $.36^{\star \star}$ \\
\hline Prudencia & $.35^{\star \star}$ \\
\hline Espiritualidad & $.31^{\star \star}$ \\
\hline Vitalidad & $.31^{\star *}$ \\
\hline Gratitud & $.30^{\star *}$ \\
\hline Integridad & $.30^{\star *}$ \\
\hline Persistencia & $.29^{\star \star}$ \\
\hline Bondad & $.29^{\star \star}$ \\
\hline Perspectiva & $.29^{\star \star}$ \\
\hline Autorregulación & $.28^{\star \star}$ \\
\hline Esperanza & $.28^{\star \star}$ \\
\hline Inteligencia social & $.24^{\star \star}$ \\
\hline Humildad & $.23^{\star \star}$ \\
\hline Amor & $.22^{\star \star}$ \\
\hline Curiosidad & $.22^{\star \star}$ \\
\hline Ciudadanía & $.17^{\star \star}$ \\
\hline Apreciación & $.16^{\star \star}$ \\
\hline Apertura mental & $.16^{\star *}$ \\
\hline
\end{tabular}


Tabla 3.

Correlaciones entre Fortalezas del Carácter y Deseabilidad Social (continuación)

\begin{tabular}{lc}
\hline Variable & EDSCM \\
\hline Creatividad & $.14^{\star \star}$ \\
Liderazgo & $.14^{\star \star}$ \\
Humor & $.13^{\star \star}$ \\
Amor por saber & $.12^{\star \star}$ \\
Valentía & $.11^{\star \star}$ \\
\hline
\end{tabular}

Nota. EDSCM = Escala de Deseabilidad Social de Crowne y Marlowe; Apreciación = apreciación de la belleza y la excelencia

${ }^{* *} p<.01$, bilateral.

\section{Estudio 4: Validez del IVyF en Relación con un Criterio Objetivo}

Park y Peterson (2007) han propuesto que, debido a la potencial desventaja por ellos supuesta del vínculo entre deseabilidad social y autoinforme de rasgos positivos, se debería obtener información de múltiples fuentes para la evaluación de las fortalezas del carácter, una de las cuales serían los observadores informantes, i. e., familiares, amigos o compañeros que evalúen el carácter de una persona objetivo. El punto de vista de Park y Peterson es que esas múltiples informaciones obtenidas sobre las fortalezas del participante no deberían utilizarse para crear un compuesto único sino para crear una imagen compleja; los autores llaman a este procedimiento metodología 360 grados.

Por su parte, Meyer et al. (2001) en relación con el autoinforme y al informe de los observadores, han enfatizado que cualquier método único de medición provee una representación parcial o incompleta de la característica que pretende medir, y que las diferentes fuentes proveen una información única. En este sentido, se halló que los autoinformes de personalidad para adultos presentan de pequeñas a moderadas asociaciones con las mismas características medidas por observadores.

En Psicología Positiva también ha sido analizada la vinculación entre autopuntuaciones y puntuaciones de las evaluaciones de informantes. Un metaanálisis realizado sobre estudios acerca del bienestar — que incluyó los conceptos satisfacción con la vida, felicidad, afecto positivo y negativo- halló una media de correlaciones de $r=.44$ entre las puntuaciones de las autoevaluaciones y las evaluaciones de los informantes (Schneider \& Schimmack, 2009).

Específicamente, en relación con la medición de los rasgos positivos, un estudio (Ruch et al., 2010) utilizando la versión alemana del VIA-IS mostró que la autoevaluación y la evaluación realizada por observadores convergían en un rango 
esperable, siendo la mediana de las 24 fortalezas del carácter de .40 . Con relación al procedimiento de obtención de datos utilizado en el estudio de Ruch et al., la evaluación objetiva fue realizada en más del $50 \%$ de los casos por dos informantes. Los resultados de ese estudio mostraron la convergencia más elevada entre autopuntuación y puntuaciones de informante para la fortaleza religiosidad y la más baja para integridad. Ruch et al. han sostenido que las convergencias halladas en su investigación son comparables con las convergencias de las variables de personalidad, y son más elevadas que las convergencias halladas para la versión del VIA-IS de EE.UU. entre las nominaciones de fortalezas del carácter realizada por otros - amigos y familiares - y la escala correspondiente, rs $=.30$ (Peterson \& Park, 2004).

\section{Participantes}

Dos muestras de participantes voluntarios de Argentina se utilizaron para este análisis: una muestra de sujetos observados y una muestra de sujetos observadores. La muestra de los participantes que constituyó el conjunto de sujetos observados estuvo constituida por 178 personas, 99 mujeres y 79 hombres, con un promedio de edad de 32.9 años, $D T=11.6$. La muestra que constituyó el conjunto de sujetos observadores fue de 178 personas, 95 mujeres y 83 varones, cuya edad promedio fue de 32.5 años, $\mathrm{DT}=11.3$. Los observadores conocían a los sujetos observados desde hacía una media de 13 años, DT $=12.4$, y los vínculos sociales con ellos eran en su mayoría cercanos -noviazgo o amistad-, $n=97$, o familiares, $n=74$, en tanto que una minoría de participantes mantenía relaciones laborales $n=7$. Ninguno de los participantes, que fueron voluntarios, recibió retribución alguna por su participación.

\section{Instrumentos}

Se utilizó el IVyF para que los participantes observados autoinformen sus fortalezas del carácter según la clasificación y definiciones de Peterson y Seligman (2004). La consistencia interna de esta muestra fue de $\alpha=.80$.

Se utilizó el IVyF en formato informante para que los participantes que constituyeron la muestra de observadores informen los rasgos positivos de los observados. El IVyF en formato informante es idéntico al IVyF original, excepto que las instrucciones y los ítems están adaptados de modo tal que se refieren a otra persona, en vez de a uno mismo. El cálculo de consistencia interna sobre los 24 ítems del IVyF en formato informante dio como resultado un $\alpha=.85$ para esta muestra.

\section{Procedimiento y análisis de datos}

Para evitar la maximización de las asociaciones entre las puntuaciones provenientes de los observadores y de los observados, se eligió un procedimiento conservador consistente en utilizar un solo observador por sujeto, en lugar de 
varios observadores por sujeto (Meyer et al., 2001). En consecuencia, se utilizaron las puntuaciones aportadas por pares de participantes observadores-observados.

A fin de responder según los instrumentos, se siguió el siguiente procedimiento: un participante recibía una batería compuesta por los dos instrumentos mencionados en el apartado Instrumentos, en su formato de papel y lápiz, más una instrucción escrita. Esta instrucción solicitaba al participante que complete el IVyF — quedando constituido como sujeto observado - y que eligiese a una persona - familiar, pareja o amigo/a que conociese al sujeto observado desde hacía más de un año- para que complete el IVyF en formato informante cumpliendo esta última persona el papel de sujeto observador-.

Se utilizaron las correlaciones $r$ de Pearson para evaluar el grado de asociación entre las puntuaciones del IVyF y las puntuaciones del IVyF en formato informante. La significación estadística se consideró de forma bilateral.

\section{Resultados}

Las correlaciones entre las puntuaciones del autoinforme de fortalezas del carácter y las puntuaciones de las fortalezas provenientes del IVyF en formato informante presentaron una media y una mediana de .49 , respectivamente, con un rango que se extendió desde un máximo de correlación de .75 correspondiente a la fortaleza espiritualidad a un mínimo de correlación de .35 correspondiente a la fortaleza integridad (ver Tabla 4). Los valores que se hallaron en esta investigación son similares, a la vez que algo superiores, a los hallados por la investigación de Ruch et al. (2010). Particularmente, ambos resultados coinciden en las fortalezas del carácter que muestran convergencias máximas —espiritualidad y amor por el saber-, y mínima -integridad-. Con relación al IVyF, las convergencias más bajas entre las puntuaciones de las autoevaluaciones y las evaluaciones hechas por personas conocidas se encuentran en el rango de valores esperables, si se considera como punto de referencia a los valores correspondientes a las convergencias halladas para los constructos de personalidad (Meyer et al., 2001).

\section{Tabla 4.}

Correlaciones entre el Autoinforme y el Informe de Observador

\begin{tabular}{lc}
\hline Escala del IVyF & IVyF \\
\hline Espiritualidad & .75 \\
Amor por el Saber & .58 \\
Humildad & .56 \\
Amor & .55
\end{tabular}


Tabla 4.

Correlaciones entre el Autoinforme y el Informe de Observador

\begin{tabular}{lc}
\hline Escala del IVyF & IVy \\
\hline Autorregulación & .55 \\
Ciudadanía & .55 \\
Valentía & .54 \\
Persistencia & .54 \\
Apreciación & .53 \\
Prudencia & .51 \\
Imparcialidad & .51 \\
Bondad & .51 \\
Apertura Mental & .48 \\
Gratitud & .48 \\
Clemencia & .48 \\
Curiosidad & .48 \\
Liderazgo & .48 \\
Creatividad & .44 \\
Esperanza & .40 \\
Inteligencia Social & .39 \\
Perspectiva & .37 \\
Vitalidad & .37 \\
Humor & .36 \\
Integridad & .35 \\
\hline Nota Apreciación & .36
\end{tabular}

Nota. Apreciación = apreciación de la belleza y la excelencia.

\section{Discusión}

Las asociaciones halladas entre las fortalezas del carácter medidas con el IVyF y las variables que forman parte de la red conceptual del carácter como es conceptualizado por Peterson y Seligman (2004) son semejantes a lo encontrado en otras investigaciones.

En relación con la satisfacción con la vida, los resultados de este estudio coinciden de forma sustancial con los hallados por la investigación de Park et al. (2004). Las siete correlaciones parciales más elevadas con satisfacción con la vida - fortalezas del carácter gratitud, curiosidad, vitalidad, esperanza, persistencia, amor y perspectiva - son las mismas para ambas investigaciones.

En relación con las asociaciones halladas entre las fortalezas del carácter y la personalidad según el Modelo de los Cinco Grandes, los resultados de esta investigación vuelven a respaldar la propuesta por Peterson y Seligman (2004) de 
que las fortalezas del carácter están asociadas a los rasgos de personalidad del Big Five. Siguiendo su argumentación, los hallazgos empíricos de esta investigación vuelven a mostrar que la clasificación de rasgos positivos VIA tiene sentido.

Los resultados de la investigación empírica son muy similares a los resultados empíricos de la relación entre las fortalezas del carácter de la clasificación VIA y el Big Five mostrados por Peterson y Park (2004), y a los resultados hallados por Macdonald et al. (2008). En particular, todas las asociaciones informadas por Peterson y Park entre las fortalezas del carácter y el Big Five se hallaron en esta investigación empírica. Además, en general, cada grupo de fortalezas del carácter que se hallaron vinculadas a cada factor del Big Five en la investigación de Macdonald et al. (2008), se encontraron también vinculadas en esta investigación empírica.

Por su parte, varias fortalezas del carácter están asociadas a más de un factor del Big Five, al igual que lo hallado en el estudio de Macdonald et al. (2008). En este sentido, los resultados respaldan la sugerencia de los autores mencionados previamente de que la propuesta de Peterson y Seligman de que cada fortaleza del carácter estaría representada por un constructo del Big Five está puesta en cuestión. Sin embargo, el examen de ciertos puntos en común entre los hallazgos del estudio de Macdonald et al. y esta investigación parecería sostener parcialmente la idea de Peterson y Seligman de vinculación biunívoca entre factores del Big Five y fortalezas del carácter. Al restringir el examen de los resultados de esta investigación y de la investigación empírica de Macdonald et al. a las vinculaciones más fuertes entre los factores del Big Five y las fortalezas del carácter, surgen coincidencias sugerentes: el factor responsabilidad aparece vinculado con persistencia, vitalidad y autorregulación; el factor agradabilidad con bondad, clemencia e imparcialidad; el factor apertura a la experiencia con las fortalezas apreciación de la belleza y la excelencia, amor por el saber y curiosidad; el factor extraversión con humor, inteligencia social y ciudadanía; y el factor neuroticismo con esperanza. Este análisis acotado de las relaciones entre Big Five y fortalezas del carácter muestra a diferentes factores del Big Five vinculados a diferentes fortalezas.

Con relación a la deseabilidad social, los resultados de las investigaciones de Macdonald et al. (2008), Sarros y Cooper (2006), Osin (2009) y Ruch et al. (2010), además de los resultados de los análisis de este estudio, muestran que no puede sostenerse la afirmación de falta de asociación entre las fortalezas del carácter y deseabilidad social sugerida por Peterson y Seligman (2004). Más bien, recibe respaldo empírico el razonamiento de que si las fortalezas del carácter son socialmente deseables - como propusieron Peterson y Seligman - las puntuaciones de fortalezas deben tender, en general, a asociarse a deseabilidad social.

Con relación a la validez relacionada con observadores objetivos, los resultados indican que las puntuaciones del IVyF convergen con las evaluaciones realizadas 
por informantes dentro del rango esperable para mediciones comparables de personalidad (Meyer et al., 2001). Además, el resultado de la convergencia del IVyF es similar al hallado para el VIA-IS (Ruch et al., 2010).

Finalmente, los resultados de los análisis psicométricos del IVyF, instrumento para evaluar las fortalezas del carácter según la clasificación VIA, muestran que el inventario posee propiedades psicométricas aceptables para su utilización en investigaciones, y semejantes a las propiedades psicométricas del VIA-IS y al IPIP-VIA-r. Además, los resultados del estudio de las asociaciones entre las fortalezas del carácter medidas con el IVyF y las variables de la red conceptual de la clasificación VIA en general replicaron las asociaciones halladas por otras investigaciones que utilizaron el VIA-IS y el IPIP-VIA-r para medir fortalezas. En consecuencia, los resultados de esta investigación respaldan la utilización del IVyF para la medición de fortalezas del carácter de la clasificación VIA de Peterson y Seligman (2004) en investigaciones empíricas académicas.

\section{Limitaciones y Futuros Estudios}

Una de las limitaciones de la presente investigación sería la falta de representatividad de población no urbana argentina. En este sentido, futuros estudios podrían desarrollarse con muestras de población general rural o de pueblos originarios rurales argentinos.

Cronbach (1989) propuso que la validación es un proceso largo y tal vez sin fin. Siguiendo este concepto, se deben reconocer limitaciones que apunten a futuros estudios. Por ejemplo, un estudio podría analizar la validez convergente entre el IVyF y otras mediciones de fortalezas del carácter, como el del VIA-IS o el IPIP-VIA-r. Además, podría investigarse la validez de grupos conocidos para cada fortaleza del carácter, p. ej., comparando artistas y no artistas para la fortaleza del carácter apreciación de la belleza y la excelencia, los religiosos y los no religiosos para la fortaleza del carácter espiritualidad, etc.

Finalmente, se han propuesto algunos métodos alternativos para calcular la confiabilidad de los instrumentos con un solo ítem por variable, como utilizar la fórmula para corrección de la atenuación (p. ej., Wanous, Reichers \& Hudy, 1997), hacer análisis factorial incorporando los ítems de los instrumentos largos homólogos (p. ej., Denissen, Geenen, Selfhout, \& Van Aken, 2008; Wanous \& Hudy, 2001; Woods \& Hampson, 2005) o usar modelación lineal jerárquica incorporando evaluaciones hechas por diversos observadores (Denissen et al., 2008). En consecuencia, futuros estudios podrían focalizarse en realizar otros procedimientos para el cálculo de la confiabilidad del IVyF. 


\section{Referencias}

Castro Solano, A. (2000). Estilos de personalidad, objetivos de vida y satisfacción vital. Un estudio comparativo con adolescentes argentinos (Tesis Doctoral). Universidad Complutense de Madrid, España.

Castro Solano, A., \& Casullo, M. M. (2001). Rasgos de personalidad, bienestar psicológico y rendimiento académico en adolescentes argentinos. Interdisciplinaria Revista de Psicología y Ciencias Afines, 18(1), 65-85.

Cohen, J. (1992). A power primer. Psychological Bulletin, 112(1), 155-159. doi:10.1037/0033-2909.112.1.155

Cosentino, A. C. (2009). Evaluación de las virtudes y fortalezas humanas en población de habla hispana. Psicodebate, 10, 53-71.

Cosentino, A. C., \& Castro Solano, A. (2008a). Adaptación y validación argentina de la Marlowe-Crowne Social Desirability Scale. Interdisciplinaria Revista de Psicología y Ciencias Afines, 25(2), 197-216.

Cosentino, A. C., \& Castro Solano, A. (2008b). Inventario de virtudes y fortalezas. Manuscrito sin publicar.

Cronbach, L. J. (1989). Construct validation after thirty years. En R. E. Linn (Ed.), Intelligence: Measurement, theory, and public policy (pp. 147-171). Urbana: University of Illinois Press.

Crowne, D. P., \& Marlowe, D. (1964). The approval motive: Studies in evaluative dependence. New York, NY, US: John Wiley \& Sons.

Dahlsgaard, K., Peterson, C., \& Seligman, M. E. P. (2005). Shared virtue: The convergence of valued human strengths across culture and history. Review of General Psychology, 9(3), 203-213. doi:10.1037/1089-2680.9.3.203

Denissen, J.J.A., Geenen, R., Selfhout, M. \& Van Aken, M.A.G. (2008). Single-item big five ratings in a social network design. European Journal of Personality, 22, 37-54.

Diener, E., Emmons, R. A., Larsen, R. J., \& Griffin, S. (1985). The Satisfaction With Life Scale. Journal of Personality Assessment, 49(1), 71-75. doi:10.1207/ s15327752jpa4901_13 
Gable, S. L., \& Haidt, J. (2005). What (and why) is positive psychology? Review of General Psychology, 9(2), 103-110. doi:10.1037/1089-2680.9.2.103

Goldberg, L. R. (1993). The structure of phenotypic personality traits. American Psychologist, 48(1), 26-34. doi:10.1037/0003-066X.48.1.26

Goldberg, L. R. (1999). A broad-bandwidth, public-domain, personality inventory measuring the lower-level facets of several five-factor models. En I. Mervielde, I. Deary, F. De Fruyt, \& F. Ostendorf(Eds.), Personality psychology in Europe (Vol. 7, pp. 7-28). Tilburg, Países Bajos: Tilburg University Press.

Goldberg, L. R., Johnson, J. A., Eber, H. W., Hogan, R., Ashton, M. C., Robert, C., \& Gough, H. G. (2006). The International Personality Item Pool and the future of public-domain personality measures. Journal of Research in Personality, 40(1), 84-96. doi:10.1016/j.jrp.2005.08.007

Helms, J. E., Henze, K. T., Sass, T. L., \& Mifsud, V. A. (2006). Treating Cronbach's Alpha Reliability Coefficients as Data in Counseling Research. The Counseling Psychologist, 34(5), 630-660. doi:10.1177/0011000006288308

Linley, P. A., Maltby, J., Wood, A. M., Joseph, S., Harrington, S., Peterson, C., ... Seligman, M. E. P. (2007). Character strengths in the United Kingdom: The VIA Inventory of Strengths. Personality and Individual Differences, 43(2), 341-351. doi:10.1016/j.paid.2006.12.004

Macdonald, C., Bore, M., \& Munro, D. (2008). Values in action scale and the Big 5: An empirical indication of structure. Journal of Research in Personality, 42(4), 787-799. doi:10.1016/j.jrp.2007.10.003

Meyer, G. J., Finn, S. E., Eyde, L. D., Kay, G. G., Moreland, K. L., Dies, R. R., ... Reed, G. M. (2001). Psychological testing and psychological assessment: A review of evidence and issues. American Psychologist, 56(2), 128-165. doi:10.1037/0003-066X.56.2.128

Osin, E. N. (2009). Social desirability in positive psychology: bias or desirable sociality? En T. Freire (Ed.), Understanding positive life: Research and practice on positive psychology (pp. 421-442). Lisboa, Portugal: Climepsi Editores.

Park, N., \& Peterson, C. (2007). Methodological issues in positive psychology and the assessment of character strengths. En A. D. Ong \& M. H. M. van (Eds.), Oxford handbook of methods in positive psychology (pp. 292-305). New York, NY, US: Oxford University Press. 
Park, N., Peterson, C., \& Seligman, M. E. P. (2004). Strengths of character and well-being. Journal of Social and Clinical Psychology, 23(5), 603-619. doi:10.1521/jscp.23.5.603.50748

Peterson, C., \& Park, N. (2004). Classification and Measurement of Character Strengths: Implications for Practice. En P. A. Linley S. Joseph (Ed.), Positive psychology in practice (pp. 433-446). Hoboken, NJ, US: John Wiley \& Sons Inc.

Peterson, C., \& Seligman, M. E. P. (2004). Character strengths and virtues: A handbook and classification. New York, NY, US: Oxford University Press.

Peterson, C., Park, N., Pole, N., D’Andrea, W., \& Seligman, M. E. P. (2008). Strengths of character and posttraumatic growth. Journal of Traumatic Stress, 21(2), 214-217. doi:10.1002/jts.20332

Ruch, W., Proyer, R. T., Harzer, C., Park, N., Peterson, C., \& Seligman, M. E. P. (2010). Values in Action Inventory of Strengths (VIA-IS): Adaptation and validation of the German version and the development of a peer-rating form. Journal of Individual Differences, 31(3), 138-149. doi:10.1027/1614-0001/a000022

Sarros, J. C., \& Cooper, B. K. (2006). Building character: A leadership essential. Journal of Business and Psychology, 21(1), 1-22. doi:10.1007/s10869-0059020-3

Schneider, L., \& Schimmack, U. (2009). Self-informant agreement in wellbeing ratings: A meta-analysis. Social Indicators Research, 94(3), 363-376. doi:10.1007/s11205-009-9440-y

Seligman, M. E. P., \& Csikszentmihalyi, M. (2000). Positive psychology: An introduction. American Psychologist, 55(1), 5-14. doi:10.1037/0003066X.55.1.5

Wanous, J.P. \& Hudy, M.J. (2001). Single-item reliability: A replication and extension. Organizational Research Methods, 4, 361-375.

Wanous, J.P., Reichers, A.E. \& Hudy, M.J. (1997). Overall job satisfaction: How good are single-item measures? Journal of Applied Psychology, 82, 247-252.

Woods, S.A. \& Hampson, S.E. (2005). Measuring the Big Five with single items using a bipolar response scale. European Journal of Personality, 19, 373-390. 